\title{
Transformer Model in Wide Frequency Bandwidth for Power Electronics Systems
}

\author{
Carlos Gonzalez-Garcia and Jorge Pleite \\ Electronic Technology Department, Carlos III University of Madrid, Avenida de la Universidad 30, Leganés, 28911 Madrid, Spain \\ Correspondence should be addressed to Carlos Gonzalez-Garcia; cggphd@gmail.com
}

Received 1 June 2012; Revised 30 October 2012; Accepted 30 November 2012

Academic Editor: Francesco Profumo

Copyright (C) 2013 C. Gonzalez-Garcia and J. Pleite. This is an open access article distributed under the Creative Commons Attribution License, which permits unrestricted use, distribution, and reproduction in any medium, provided the original work is properly cited.

\begin{abstract}
The development of the smart grids leads to new challenges on the power electronics equipment and power transformers. The use of power electronic transformer presents several advantages, but new problems related with the application of high frequency voltage and current components come across. Thus, an accurate knowledge of the transformer behavior in a wide frequency range is mandatory. A novel modeling procedure to relate the transformer physical behavior and its frequency response by means of electrical parameters is presented. Its usability is demonstrated by an example where a power transformer is used as filter and voltage reducer in an AC-DC-AC converter.
\end{abstract}

\section{Introduction}

The future trends on the power delivery are focused on the development of a smart grid. This concept implies new challenges as interconnection or treatment of renewable energy resources and the application of technologies such as telecommunication or power electronics whose products and solutions should play an important role in the future of the medium voltage distribution.

The power transformer is widely spread in electrical systems providing basic functionalities such as voltage insulation and voltage adaptation. As an essential element on the energy distribution, it must be involved in the new smart grid perspective, and therefore new roles regarding its manufacturing, maintenance, use, and operation must be taken into account. One of these roles consists in the analysis of its behavior in a wide frequency bandwidth, not limited to the 50 or $60 \mathrm{~Hz}$ power frequency.

Considering the transformer maintenance program, most of routine tests to assess the transformer condition are based on power frequency $(50 / 60 \mathrm{~Hz})$ measurements (load and no load losses, capacitance, and Tan-Delta). However, the FRA test measures the impedance between two terminals of the transformer in the $20 \mathrm{~Hz}$ to $20 \mathrm{MHz}$ bandwidth. It has been extensively proven that this procedure allows the detection of failures that are not visible for other techniques [1].

Considering the power delivery, the conventional linefrequency transformers are not able to deal with power quality problems (e.g., sags, swells, flicker, and harmonics). To solve this task, there have been some attempts (although still under development) for the installation of additional equipment as power electronics converter that operates at higher switching frequencies in the medium voltage grid. It is also applied in the LV grid due to the advances in semiconductor technology (faster switching actions, higher blocking voltages, and higher power densities) and the development of new magnetic materials with low loss densities at higher operating frequencies $[2,3]$. The application of PETs is extensible to traction applications [4] or Plug-in Electrical Vehicle (PEV) charging stations [5].

This solution implies replacing the typical power frequency distribution transformer by a high frequency switched one, commonly named as PET or Intelligent SolidState Transformer (ISST) consisting in a Medium Frequency Transformer (MFT) and one or several power electronic converters. As a result, fast switching actions are applied directly to transformer terminals, so that overall magnetic volume is reduced and more compact converter designs are 


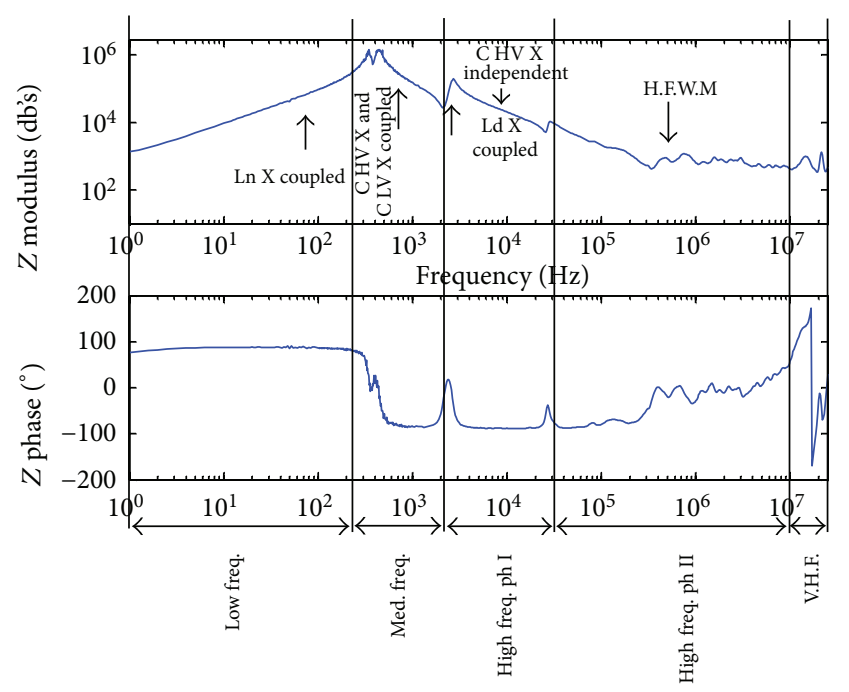

FIGURE 1: Identification of the most significant bandwidths in an end-to-end open circuit typical response.

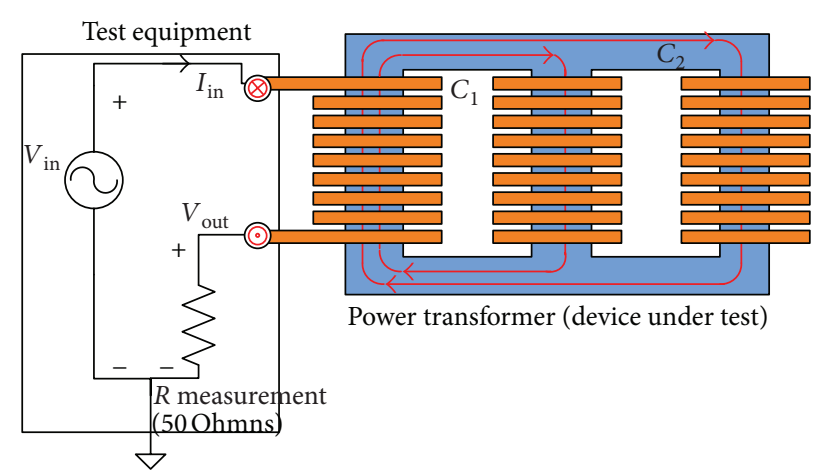

FIGURE 2: Electromagnetic field distribution at low frequency bandwidth. Mainly magnetic on the core.

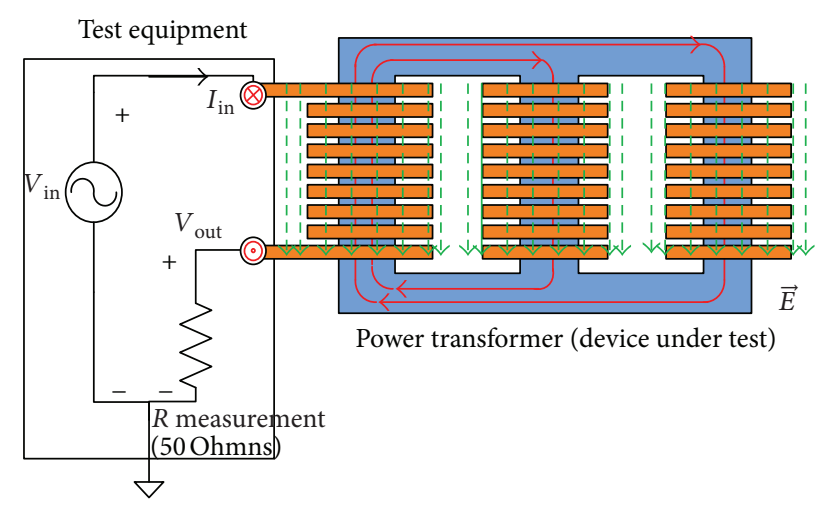

FIGURE 3: Electromagnetic field distribution at medium frequency bandwidth. Mainly electric field along the windings $E$.

reached [6]. However, new problems regarding losses or overheating and subsequent insulation damages and loss of transformer life expectancy could come across. Therefore, an accurate knowledge of the transformer in a wider frequency bandwidth is needed to tackle with new analysis.

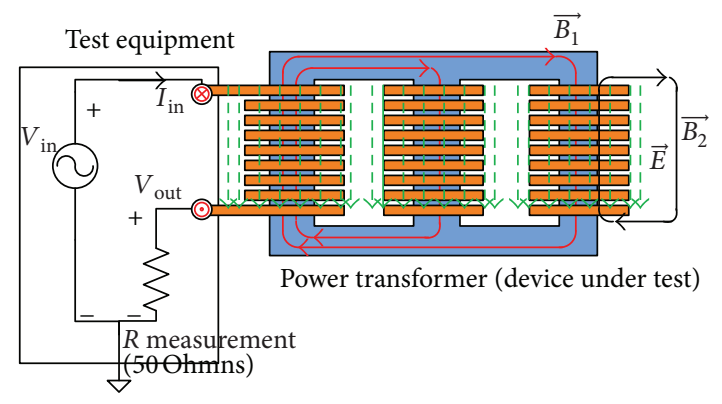

FIGURE 4: Electromagnetic field distribution at high frequency phase I bandwidth. Coexistence of electric field along the windings $E$, and magnetic field on core $B_{1}$ and core-dielectric $B_{2}$.

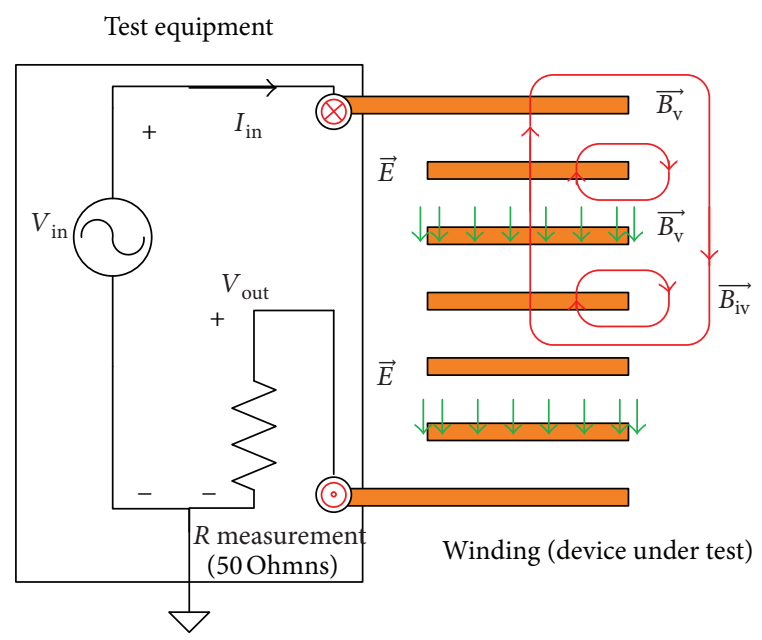

FIGURE 5: Electromagnetic field distribution at high frequency phase II bandwidth. Coexistence of electric $E$ and magnetic $B_{v}, B_{i v}$ field exclusively along the measured winding.

Transformer models have been used to interpret and analyze the transformer behavior, but they have been commonly limited to a narrow bandwidth centered in the power frequency.

In this paper, a novel procedure to obtain transformer models, that are able to represent the transformer behavior in a wide frequency bandwidth, is presented and applied to a $25 \mathrm{kVA} 16 \mathrm{kV} / 420 \mathrm{~V}$ YNYn distribution transformer. The accuracy of the model is evaluated comparing the simulation and actual responses, obtained by an FRA test.

Finally, the transformer model is used as part of an ACDC-AC power electronic converter. The exact knowledge of the frequency behavior allows taking advantage of the filtering characteristics associated with the leakage inductance and shunt capacitance of the windings, saving the use of the external LC filter in the final stage of the 3-phase setup.

\section{Modeling Procedure}

The proposed solution to obtain a wide frequency model is based on a novel modeling procedure developed in [7] whose main features are as follows. 


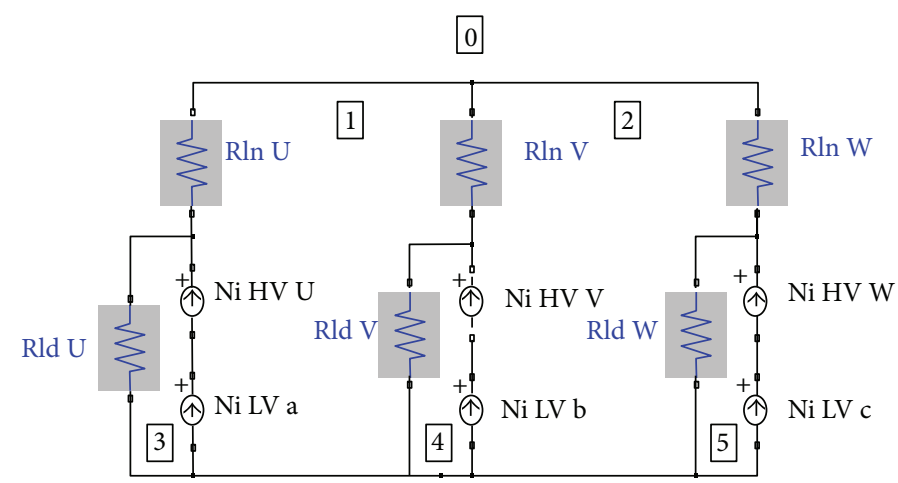

FIGURE 6: Magnetic circuit based on reluctances and magnetomotive forces for representing the magnetic field behaviour in the core and core-dielectric at low and high frequency phase I bandwidths.

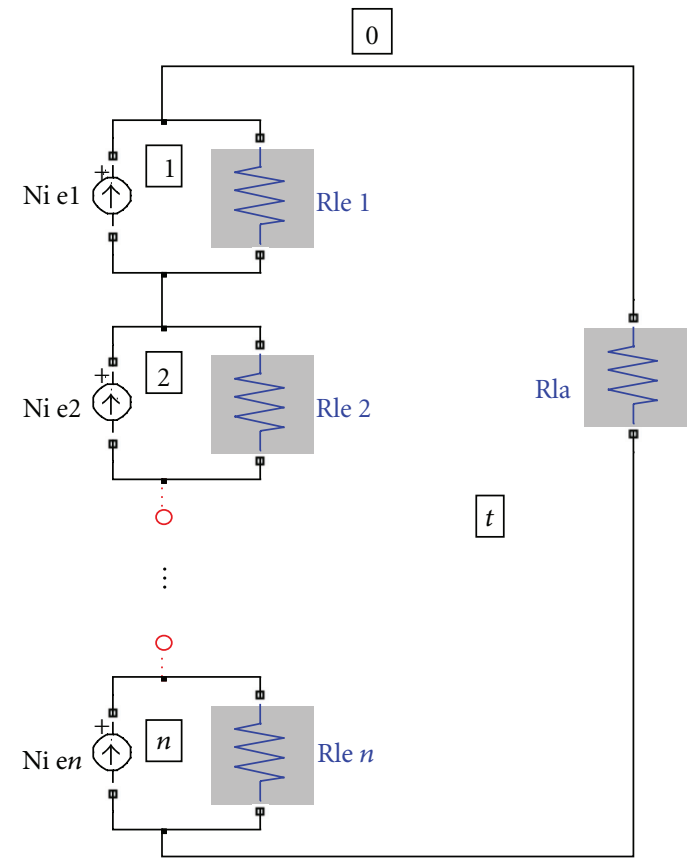

FIGURE 7: Magnetic circuit based on reluctances and magnetomotive forces for representing the magnetic field behaviour in the winding at high frequency phase II bandwidth.

(i) The input data are obtained by the Frequency Response measured in a wide frequency bandwidth on the FRA test.

(ii) The resulting model is based on a parametric structure developed using the Magnetic-Electric Duality Principle and Energy Storage Concept. These synthesizing tools allow the interpretation of the electromagnetic phenomena occurring not only on the magnetic core as other solutions proposed but also on the leakage path and dielectric media along the winding. Subsequently, the physical behavior is concentrated on an electrical circuit.

(iii) The structure is built in a modular way and constituted by plugging in different and independent blocks representing only the physical constitution of any internal element. The vector group connection of the windings (Star or Delta) is externally implemented on the model and is independent of the physical submodels or blocks.

(iv) The value of the parameters is calculated using an ad hoc optimization algorithm. It searches the solution that minimizes the difference between simulated and actual measured data. The algorithm is separately applied to the different blocks building the complete model and responsible for the response in a specific frequency bandwidth of the entire measurement sweep.

As a result, the modeling procedure follows three stages: the structure design (stage 1, Section 2.1), the algorithm design for calculation of the value of the parameters (stage 2, Section 2.2), and experimental validation (stage 3, Section 2.3)

2.1. Structure Design. The design of the structure is the first stage on the modeling procedure and it follows, for its part, other three steps.

The first one is the qualitative analysis of the electromagnetic field at different frequency bandwidths in the FRA test, divided in the partial bandwidths of Figure 1 and named as low frequency bandwidth, medium frequency bandwidth, and high frequency phase I and II bandwidths.

The constitution of the field at the low frequency bandwidth is depicted in Figure 2, where the measurement setup is included and the magnetic flux paths are represented by the $C_{1}$ and $C_{2}$ solid lines. The medium frequency bandwidth is defined as the range where the constitution of the analyzed field is mainly electrical (represented with the E dashed lines) and distributed along a structure defined along the windings (not only in the excited one but also in the rest due to the magnetic coupling) in the fashion depicted in Figure 3 with dashed lines.

The electromagnetic effects in the high frequency phase I and phase II bandwidths defined in the modeling procedure are represented in Figures 4 and 5, respectively. 


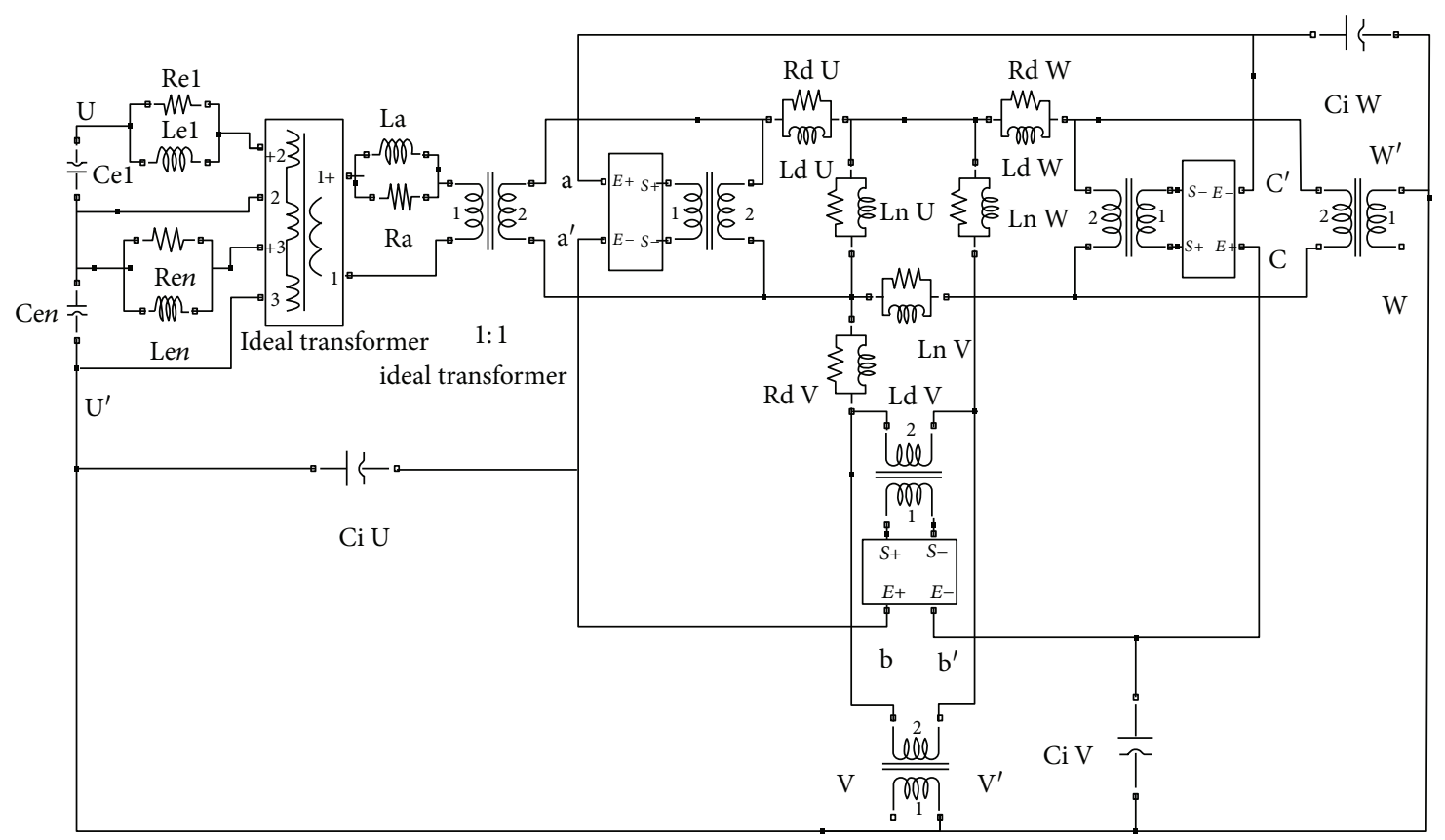

FIGURE 8: Complete electric circuit. Interwinding capacitances $\mathrm{Ci} \mathrm{U,} \mathrm{Ci} \mathrm{V,} \mathrm{and} \mathrm{Ci} \mathrm{W} \mathrm{added.}$

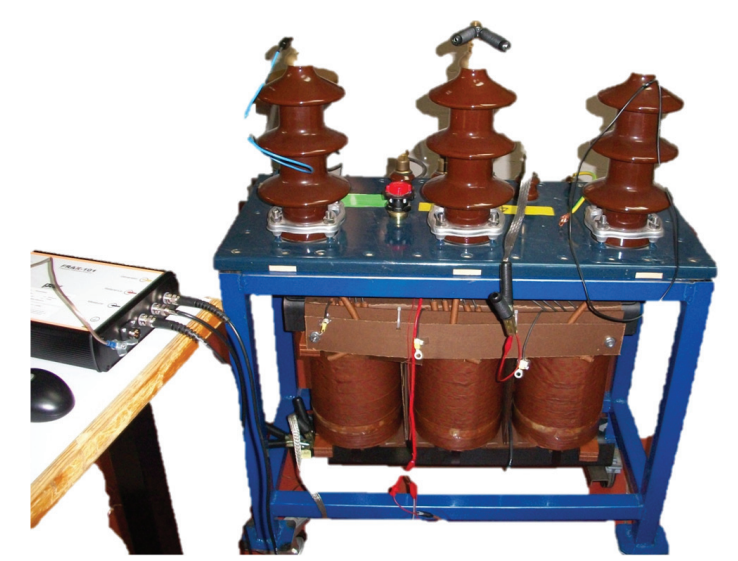

FIGURE 9: 25 kVA 16.125 kB/420 V YNyn distribution transformer measured and modeled.

The second step designing the structure consists on combining the obtained results in a magnetic circuit that simulates the same magnetic field distribution.

The referenced magnetic field in the core $\left(B_{1}\right.$ in Figure 4$)$ and in the core-dielectric $\left(B_{2}\right.$ in Figure 4$)$ for low and high frequency phase I bandwidths can be represented by the well-known magnetic circuit of Figure 6 . The parameters $\mathrm{R} \ln \mathrm{X}$ (where $\mathrm{X}$ is one of the $\mathrm{U}, \mathrm{V}$, or $\mathrm{W}$ phase of the 3 phase transformers indistinctly) represent the reluctances of the magnetic paths in the core and therefore depending on the core geometry and its permeability $\mu_{c}$. The parameters Rld X represent the reluctances of the magnetic paths in
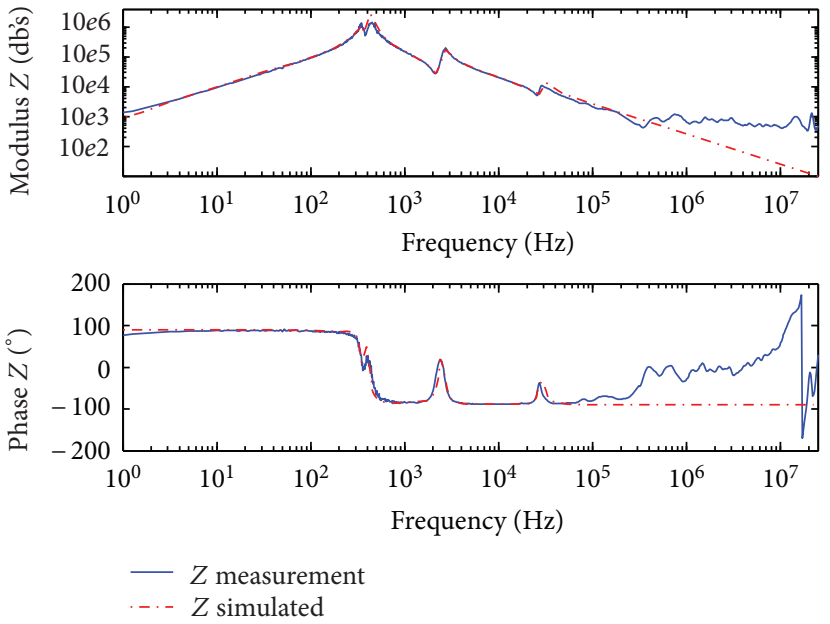

FIGURE 10: Actual versus simulated measurement. YNyn configuration end-to-end open circuit, phase U HV.

the core-dielectric and depending on the winding geometry and mainly the dielectric permeability $\mu_{d}$. The sources $\mathrm{Ni}$ $\mathrm{HV} \mathrm{X}$ and $\mathrm{Ni}$ LV $\mathrm{X}$ represent the magnetomotive forces of the HV and LV windings, respectively. Using the same representation, the magnetic circuit depicted on Figure 7 represents the magnetic field distribution for the defined high frequency phase II bandwidth, represented in Figure 5, where Rla represents the reluctance of the magnetic path of the dielectric surrounding the $N$ turns of the winding. $\mathrm{Rle}_{j}$ represents the same reluctance but for a group of $j$ turns in the 

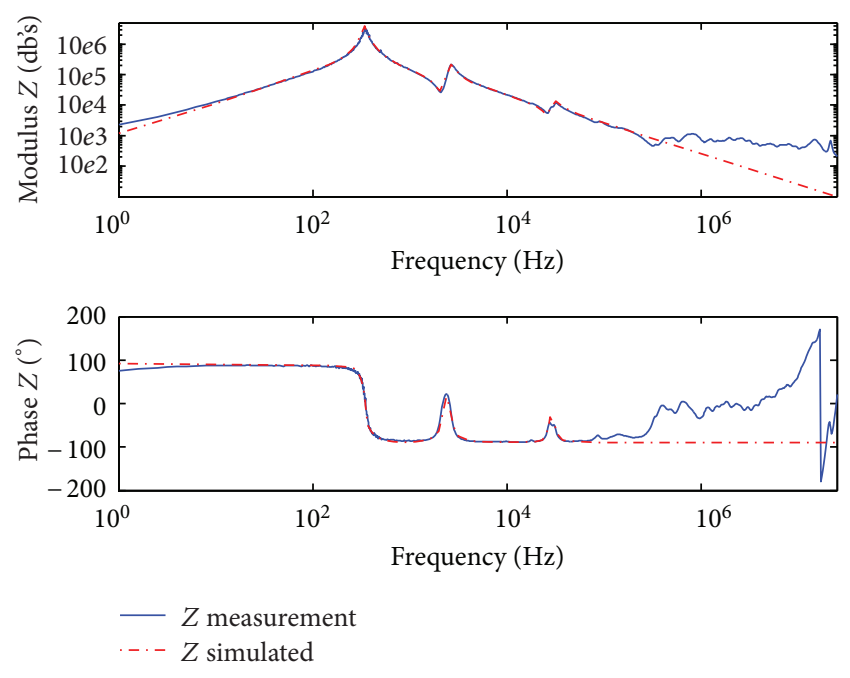

FIGURE 11: Actual versus simulated measurement. YNyn configuration end-to-end open circuit, phase V HV.

winding. Finally, $\mathrm{Nie}_{j}$ represents the magnetomotive force of a group of $j$ turns in the winding.

The third step designing the structure consists in obtaining the final equivalent electric circuit using the wellestablished Magnetic-Electric Duality Principle to convert the magnetic circuit into electric one and the Energy Storage Concept, adding $R$ and $C$ elements to represent the losses and electric field effect, respectively. The resulting three submodels are

(i) LFCM submodel representing the magnetic core, at the low frequency bandwidth of Figure 1;

(ii) HFCM submodel representing the core and dielectric media at the high frequency phase I bandwidth of Figure 1;

(iii) HFWM submodel representing the winding at the medium frequency and high frequency phase II bandwidths of Figure 1.

The complete transformer model in Figure 8 is composed of the models representing the three previous submodels (for simplicity only $\mathrm{U}$ phase for $\mathrm{HV}$ winding is completely represented with 2 groups of turns) and the capacitors $\mathrm{Ci}$ $\mathrm{X}$ that represent the electric energy storage on the dielectric media between $\mathrm{HV}$ and $\mathrm{LV}$ windings on the generic $\mathrm{X}$ phase. The YNd configuration is represented in Figure 8 to illustrate how easily the vector group is implemented only connecting the output terminals of the windings. Any of these submodels is in charge of a specific bandwidth defined in Figure 1.

2.2. Algorithm Design. The second stage of the proposed modeling procedure consists in designing the mathematical algorithm in order to fit the actual measured response by the simulation of the model. In this case, the optimization algorithm is completely developed in [7] and its working principle is summed up in the objective function given by (1).

$$
\begin{aligned}
\frac{d E_{t}}{\partial P_{i}}=\sum_{j=1}^{j=m}\left\{\left(\frac{1}{\left|Z_{\text {measured } j}\right|^{2}}\right)\right. & \left.\frac{d\left(\left|Z_{\text {submodel }}\left(\omega_{j}\right)-Z_{\text {measured } j}\right|^{2}\right)}{\partial P_{i}}\right\}=0,
\end{aligned}
$$

where $E_{t}$ represents the total error when comparing the simulated frequency response of the model and the actual response of the measured transformer, $P_{i}$ represents a generic $R, L$, or $C$ parameter of a submodel, $j$ is the index representing each frequency point in a $[1, m]$ bandwidth, $Z_{\text {measured } j}$ represents the complex impedance measured in the $j$ frequency, $Z_{\text {submodel }}\left(\omega_{j}\right)$ is the complex impedance, depending of the $R, L$, and $C$ parameters of the submodels and the angular pulsation $\omega_{j}$ for a frequency $j$.

2.3. Experimental Validation. The third and last stage in the proposed modeling procedure consists in the experimental validation of the resulting model. The model is approved if it is able to simulate the FRA measurements. For this contribution, a $25 \mathrm{kVA}, 16.125 \mathrm{kV} / 420 \mathrm{~V}$ YNyn two-winding three-phase distribution transformer (Figure 9) has been measured with an FRA commercial device and modeled by implementing the mathematical algorithm in mathemati$\mathrm{cal} /$ simulation software. The calculated parameters are shown in Table 1 where they are split using the submodel division.

Figures 10 and 11 show the comparison between the actual measurement and the simulated response of the model implemented in MATLAB Simulink.

The modeling procedure is able to produce general models with the following features.

(i) The transformer behavior in a wide frequency bandwidth is related with the parameters of the model.

(ii) The submodels are obtained based on general and common physical principles, and particularizations for specific transformers are avoided. However, if particularization needed to be considered, it could be solved with a concrete analysis of the electromagnetic field, as shown in Section 2.1.

(iii) The vector group connection is independent of the electrical structure that represents the physical behavior of the transformer. As a result, any possible vector group can be modeled by changing the terminal connections of the model. This same conclusion can be applied for any FRA measurement setup.

(iv) Modeling of $n$-windings transformer is straightforward. Only the addition of $n$ number of winding submodels is needed.

(v) Modeling of the autotransformer is straightforward. Only an external series connection of series and mutual windings is needed. 


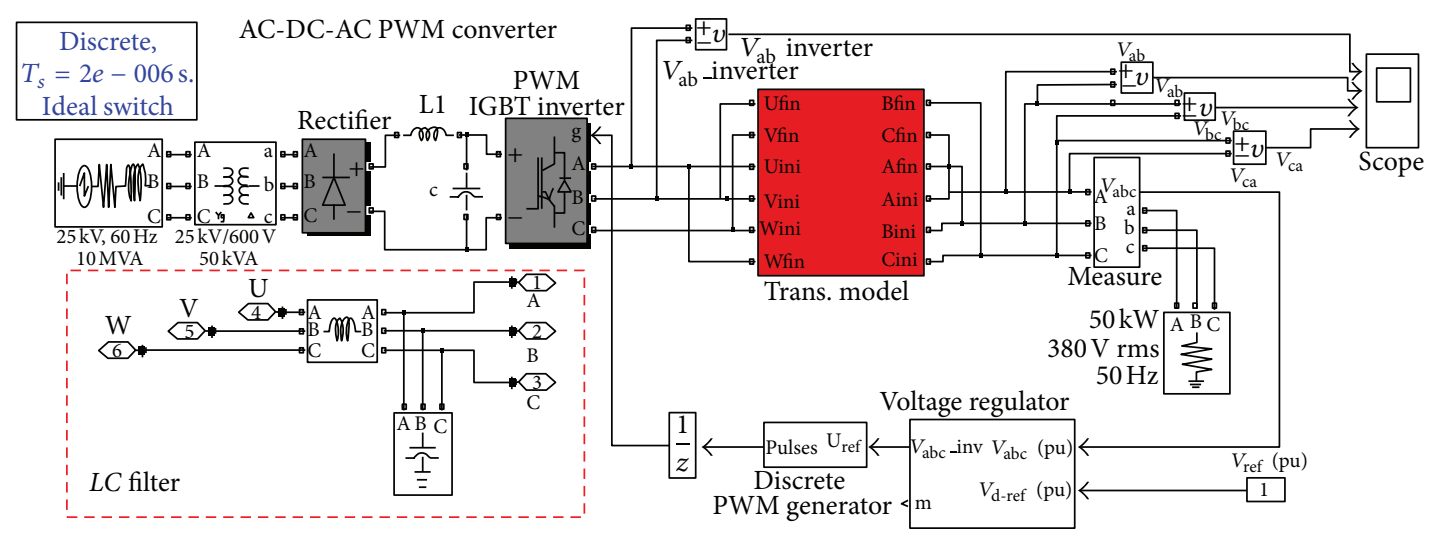

Figure 12: AC-DC-AC converter.
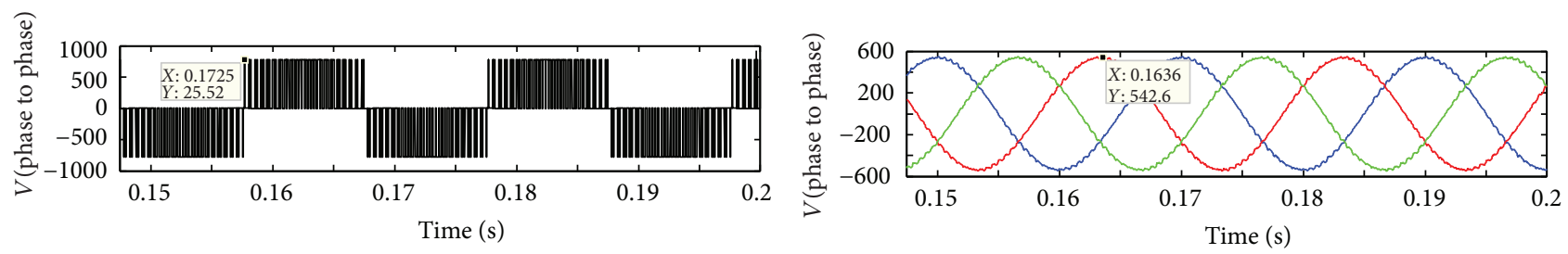

$-V_{\mathrm{ab} \_}$inverter

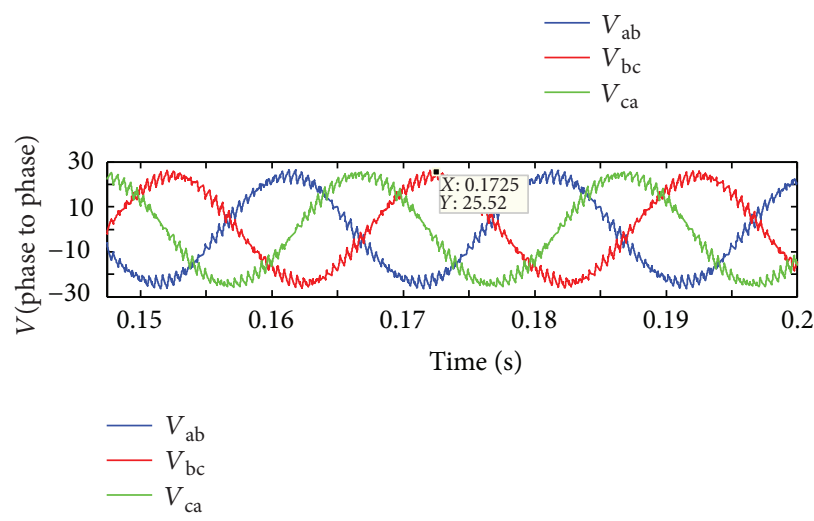

FIGURE 13: Upper (left) trace: PWM voltage; upper (right) trace: 3 phase to phase symmetrical voltages when $L C$ filter is used; lower trace: 3 phase to phase symmetrical voltages when transformer model is used.

The capability of the resulting models can be exploited for sensitivity analysis, FRA traces interpretation, and implementation of simulation systems. That allows knowing exactly the transformer behavior in a wide frequency bandwidth in order to be used in the design process of power electronics converters as it is shown in the following Section 3.

\section{AC-DC-AC Converter Application}

The DC-AC conversion needs a filter in order to obtain a sinusoidal waveform from a PWM voltage waveform, avoiding the high frequency components. This task usually can be solved with the addition of an $L C$ filter on the last stage of the converter. However, that solution implies an economic cost and total volume increase (critical for transport applications) due to the addition of $L$ and $C$ components. Moreover, an additional adaptation stage could be needed in order to increase/reduce the voltage amplitude of the inverter.

The use of a PET could solve at the same time the filtering and adaptation necessities. However, an accurate transformer model, as the one presented in previous paragraphs, is needed to assure a complete comprehension of its frequency response and therefore the behavior (losses, voltage regulator, and so on) when working as part of a DC-AC converter.

In the following example, the transformer model is implemented and simulated as part of an AC-DC-AC converter (Figure 12) based on the power electronic model named "power_bridges.mdl" in MATLAB SimPowerSystems.

The converter consists in a first stage where a DC voltage of 1000 volts is obtained. The second stage consists in a PWM inverter to obtain 3 phase to phase PWM voltages. One of the three voltages in the 3-phase converter, named as $V_{\mathrm{ab}-}$ inverter, has been plotted in the upper side of Figure 13. 
TABLE 1: Value of the parameters of the experimental model.

\begin{tabular}{|c|c|c|c|}
\hline & Phase U & Phase V & Phase W \\
\hline & \multicolumn{3}{|c|}{ L.F.C.M sub-model } \\
\hline LFB (Hz) & {$[1-2000]$} & {$[1-2000]$} & {$[1-2000]$} \\
\hline Number $^{\circ}$ of points & 201 & 201 & 201 \\
\hline $\operatorname{Ln} X(\mathrm{H})$ & 183.1504 & 399.8045 & 185.3597 \\
\hline C HV (nF) & 0.7452 & 0.6648 & 0.7356 \\
\hline \multirow[t]{2}{*}{$\operatorname{Rn} X(M \Omega)$} & 2.400 & 12.124 & 2.3 \\
\hline & \multicolumn{3}{|c|}{ H.F.C.M sub-model } \\
\hline HFPIB (kHz) & {$[2-2.89]$} & {$[2-2.89]$} & {$[2-2.89]$} \\
\hline Number of points & 17 & 17 & 17 \\
\hline $\operatorname{Ld} X(\mathrm{H})$ & 4.0227 & 5.5128 & 5.0427 \\
\hline \multirow[t]{2}{*}{$\operatorname{Rd} X(k \Omega)$} & 11.690 & 10.154 & 11.690 \\
\hline & \multicolumn{3}{|c|}{ H.F.W.M sub-model (all phases HV side) } \\
\hline HFPIIB (kHz) & {$[10-335]$} & {$[10-335]$} & {$[10-335]$} \\
\hline Number of points & 239 & 239 & 239 \\
\hline $\mathrm{Le}_{j}, j=1(\mathrm{mH})$ & 3.9218 & 3.8539 & 3.4211 \\
\hline $\mathrm{Ce}_{j}, j=1(\mathrm{nF})$ & 1.0838 & 1.0602 & 1.0721 \\
\hline $\operatorname{Re}_{j}, j=1(\mathrm{k} \Omega)$ & 7.412 & 7.895 & 7.296 \\
\hline $\mathrm{Le}_{j}, j=2(\mathrm{mH})$ & 3.9414 & 4.0031 & 3.9612 \\
\hline $\mathrm{Ce}_{j}, j=2(\mathrm{nF})$ & 2.5919 & 2.5219 & 2.5528 \\
\hline $\operatorname{Re}_{j}, j=2(\mathrm{k} \Omega)$ & 5.339 & 5.762 & 5.391 \\
\hline \multirow[t]{2}{*}{$\mathrm{C} \mathrm{HV} \mathrm{(nF)}$} & 0.7452 & 0.6648 & 0.7356 \\
\hline & \multicolumn{3}{|c|}{ Ci X parameters } \\
\hline Bandwidth (Hz) & [4083.48-10023.8] & [4083.48-10023.8] & [4083.48-10023.8] \\
\hline Number of points & 39 & 39 & 39 \\
\hline Ci X $(n F)$ & 0.1186 & 0.1170 & 0.1181 \\
\hline
\end{tabular}

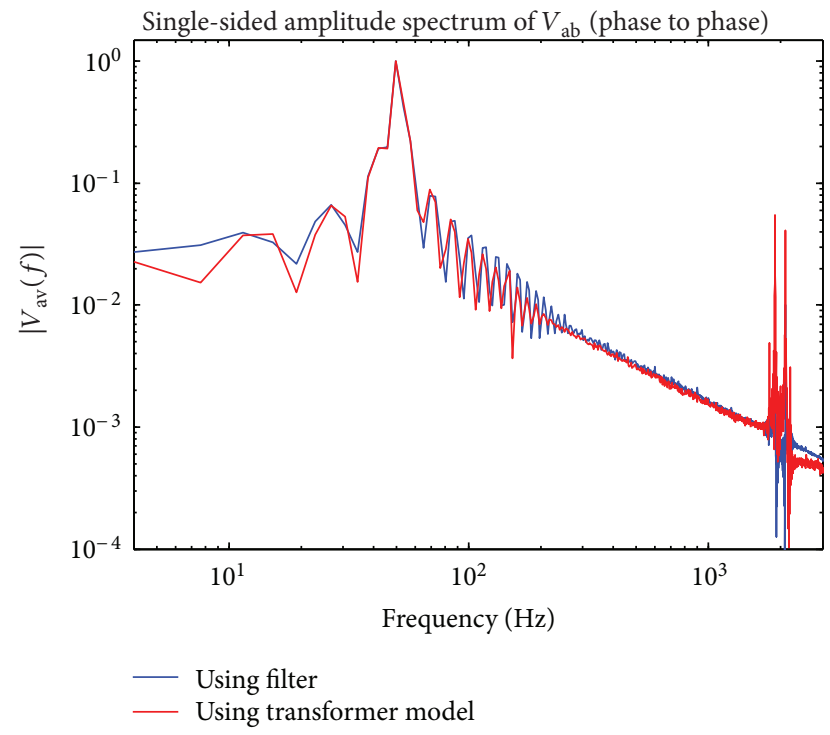

Figure 14: Spectrum of the $V_{\mathrm{ab}}$ voltages for $L C$ and transformer simulation.

The filtering and adaptation stages have been solved using two alternatives. The first one is an $L C$ filter (pointed by red dashed lines in Figure 12) used to get symmetrical 3 phase to phase sinusoidal voltages $V_{\mathrm{ab}}, V_{\mathrm{bc}}$, and $V_{\mathrm{ca}}$. The second one is a power electronic transformer that has been simulated using the presented model.

The plots of Figure 13 show the PWM voltage signal in the upper side (the input of the $L C$ or PET) and the comparison of the filtered voltage signals using the $L C$ filter (results in the middle side of Figure 13) and the model of the PET (results in the lower side of Figure 13).

It has been possible to simulate the PET because its behavior is completely represented till $500 \mathrm{kHz}$ using the model shown in Section 2. The analysis of the model (completely represented in Figure 8) and its simulated response (showed in Figures 10 and 11) demonstrate that the leakage inductance (Ld X in Table 1) and the shunt capacitance of the windings $\left(C_{\mathrm{HV}}\right.$ in Table 1$)$ conform an $L C$ filter whose values give a resonant frequency of $2600 \mathrm{~Hz}$. That means that the transformer filter can be used as the element needed in the inverter, saving the use of the external $L C$ component. This concept is developed in [8] where the bases for designing $L C$ filters integrated in the transformer is explained. The accurate agreement between actual and simulated responses until $500 \mathrm{kHz}$ demonstrates that the simulation bandwidth is enough to cover the $2600 \mathrm{~Hz}$ behavior when the cut-off frequency of the filter appears.

The use of the transformer in the complete system in spite of the $L C$ filter offers not only the filtering capability but also the voltage adaptation. For this reason, the 3 phase to phase voltages $V_{\mathrm{ab}}, V_{\mathrm{bc}}$, and $V_{\mathrm{ca}}$ (represented in the lower side of Figure 13) present a sinusoidal shape with 18 Volts $\mathrm{rms}$ in 
spite of the 383 Volts rms obtained when the external $L C$ filter is used.

The filtering capability of the transformer is demonstrated in the comparison of Figure 14. The frequency spectrum of the $V_{\mathrm{ab}}$ phase to phase sinusoidal voltage is shown for both cases, when the $L C$ and the transformer model are used. Both graphics are fairly equal, which demonstrates that the transformer filtering capability can be considered as accurate as the $L C$ filter.

Finally, the efficiency and voltage regulation of the PET can be both calculated and simulated. The calculation can be done because the values of the resistive parameters representing the losses for the complete frequency bandwidth in the model are known, as represented in the example of Table 1 in the $\mathrm{Rn} \mathrm{X}, \mathrm{Rd} \mathrm{X}, \mathrm{Re}_{j}$ parameters. As a result, an efficiency of $93.74 \%$ and a voltage regulation of $94.7 \%$ were calculated after simulation.

\section{Conclusions}

The new development of smart grids involves the use of power electronic equipment and new challenges in the operation of the power transformers. Therefore, the knowledge of its behavior in a wide frequency bandwidth, not limited to the power frequency $(50 / 60 \mathrm{~Hz})$, is a necessity.

A novel modeling procedure to obtain the transformer model in a wide frequency bandwidth is presented. The main feature of the model is its capability of interpreting the physical behavior by means of electrical parameters. That allows developing simulations, design revision, or sensitivity analysis in order to take advantages of the capabilities of the transformer in power electronics design or avoid risky situations as additional losses, overheating, or overvoltages due to high frequency components.

\section{Acronyms}

FRA: Frequency response analysis

HFCM: High frequency core model

HFWM: High frequency winding model

LFCM: Low frequency core model

PET: $\quad$ Power electronic transformers.

\section{Conflict of Interests}

The authors would like to clarify that there is not financial gain and/or commercial interest related with MEGGER and MATLAB registered trademarks. The only reason these names have been included in the paper is for the sake of precision and to determine, in the more exact way, the procedure to simulate the model and measure the prototype transformer.

\section{References}

[1] International Electrotechnical Commission, Power Transformers-Part 18: Measurement of Frequency Response, vol. 60076, International Electrotechnical Commission, Geneva, Switzerland, 2012.
[2] Z. Wang and K. Yu, "The research of Power Electronic Transformer (PET) in smart distribution network," in Proceedings of the International Conference on Power System Technology: Technological Innovations Making Power Grid Smarter (POWERCON '10), pp. 1-7, October 2010.

[3] J. M. Carrasco, L. G. Franquelo, J. T. Bialasiewicz et al., "Powerelectronic systems for the grid integration of renewable energy sources: a survey," IEEE Transactions on Industrial Electronics, vol. 53, no. 4, pp. 1002-1016, 2006.

[4] D. Dujic et al., "Laboratory scale prototype of a power electronic transformer for traction applications keywords power electronic transformer topology," in Proceedings of the 14th European Conference on Power Electronics and Applications (EPE '11), vol. 1, pp. 1-10, September 12011.

[5] P. S. Moses, M. A. S. Masoum, and K. M. Smedley, "Harmonic losses and stresses of nonlinear three-phase distribution transformers serving plug-in electric vehicle charging stations," in Proceedings of the IEEE PES Innovative Smart Grid Technologies (ISGT '11), pp. 1-6, January 2011.

[6] M. Kang, P. N. Enjeti, and I. J. Pitel, "Analysis and design of electronic transformers for electric power distribution system," IEEE Transactions on Power Electronics, vol. 14, no. 6, pp. 1133-1141, 1999.

[7] C. G. García, Procedimiento de modelado basado en el análisis de la respuesta en frecuencia y aplicación en transformadores trifásicos de potencia para su caracterización y diagnóstico. volume 1 [Ph.D. thesis], 2012.

[8] V. Valdivia, J. Pleite, P. Zumel, and C. Gonzalez, "Improving design of integrated magnetics for power electronics converters," Electronics Letters, vol. 44, no. 11, pp. 693-694, 2008. 

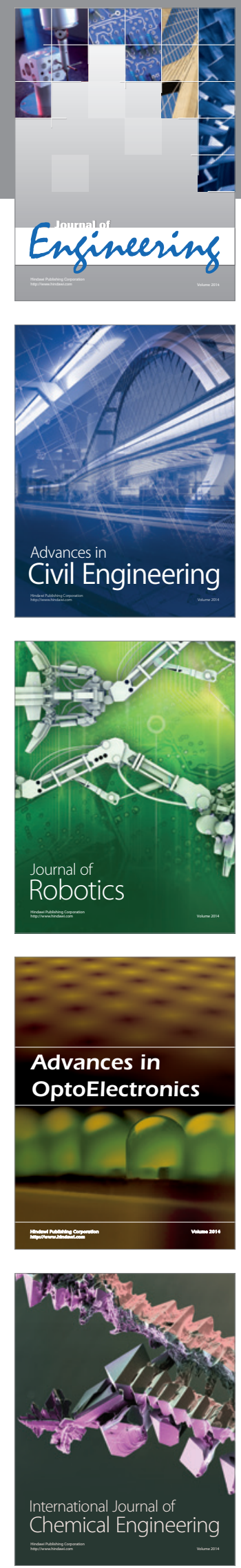

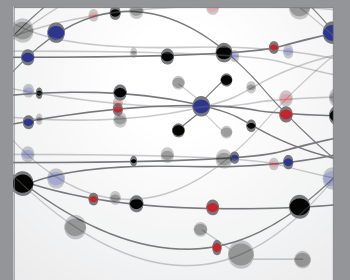

The Scientific World Journal
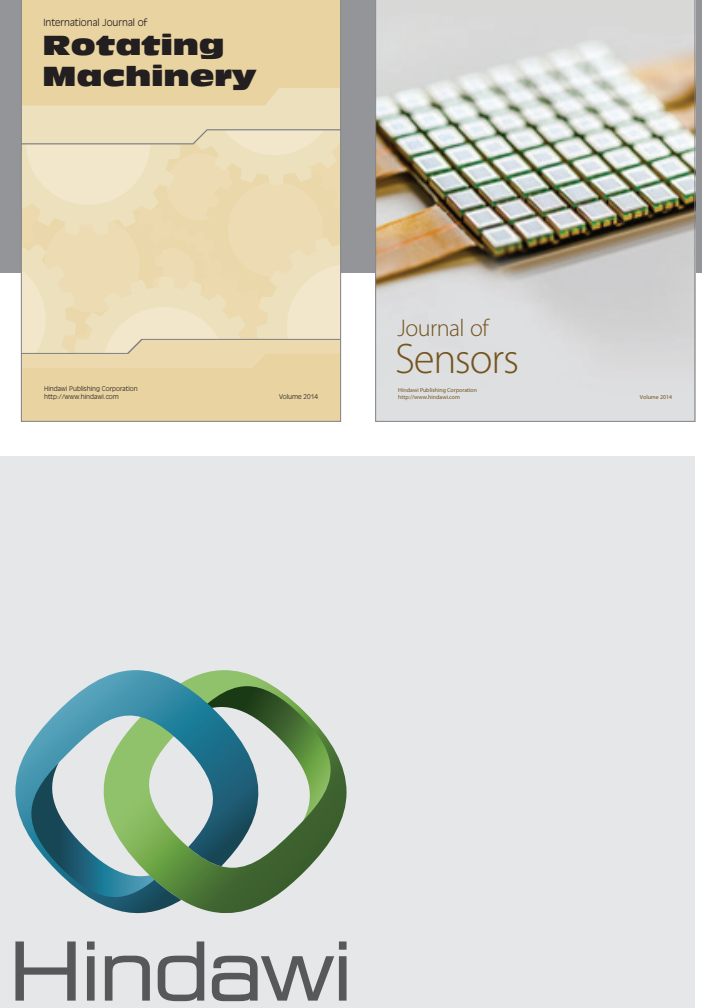

Submit your manuscripts at http://www.hindawi.com
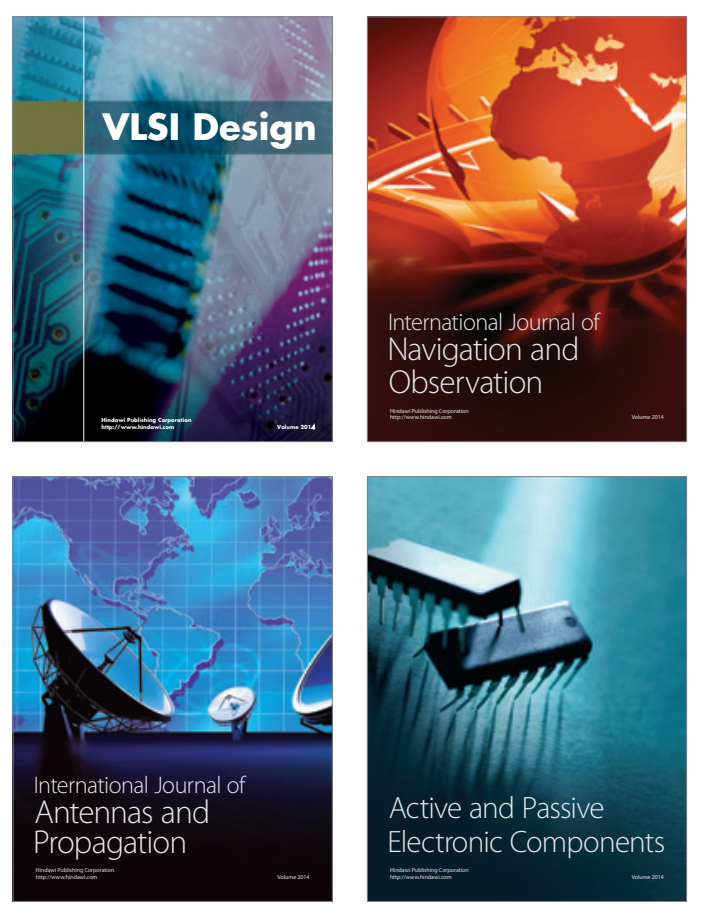
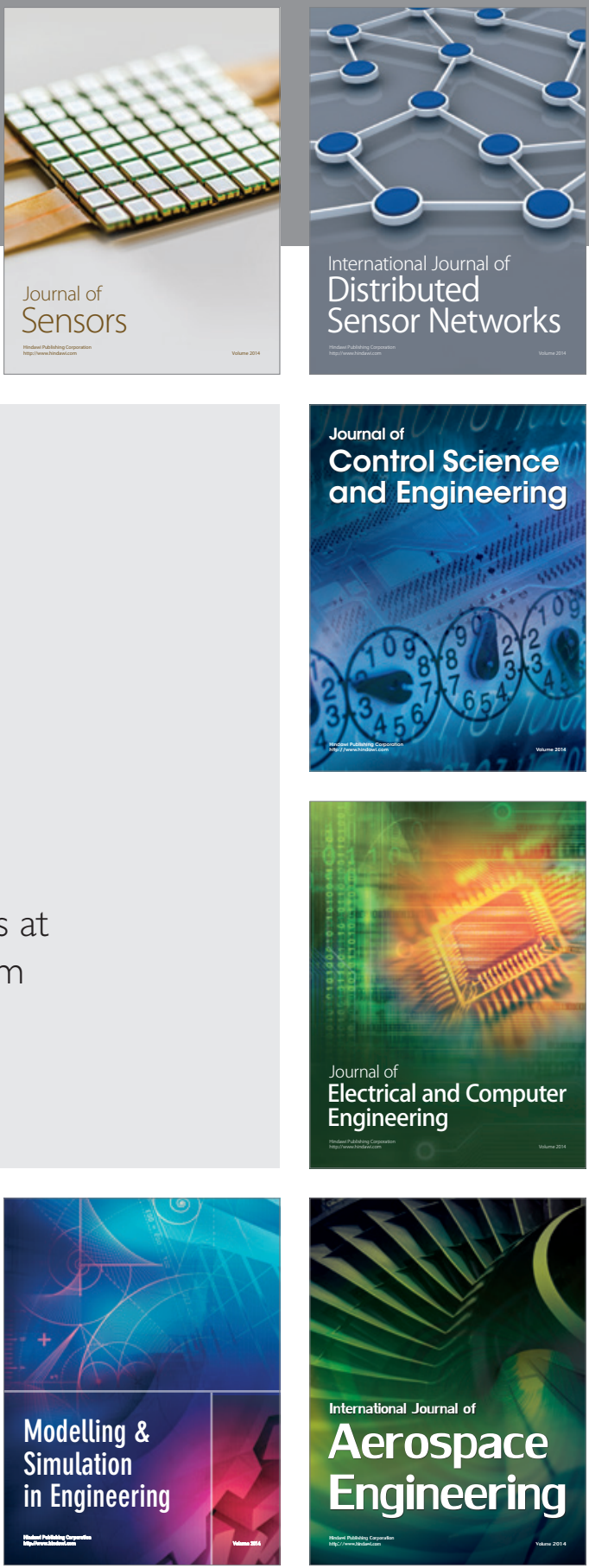

Journal of

Control Science

and Engineering
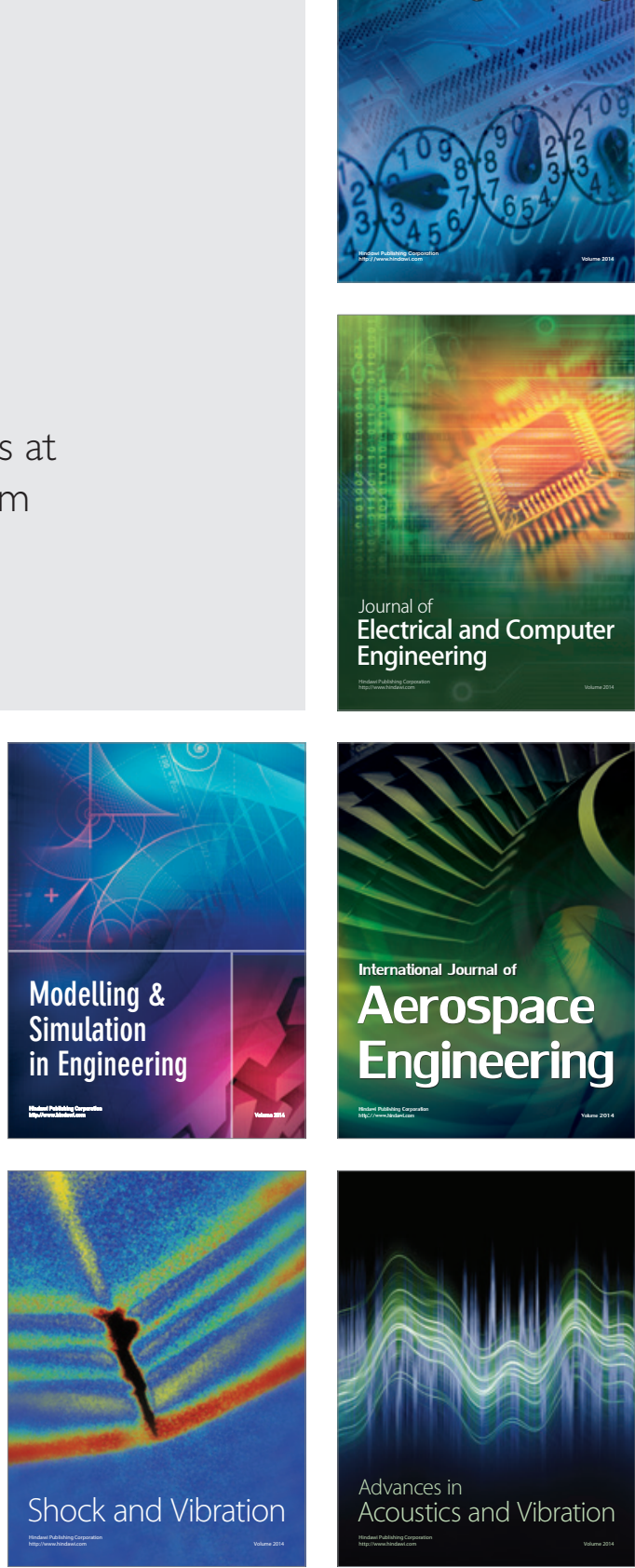